\title{
Acute Multiple Cerebral Watershed Infarctions as the Initial Presentation of COVID-19 in a Young Male Patient: A Case Report
}

\author{
Randa Abdelmasih ${ }^{1}$, Ramy Abdelmaseih ${ }^{1}$, Radhames Ramos De Oleo ${ }^{1}$ \\ 1. Internal Medicine, University of Central Florida College of Medicine, Ocala, USA
}

Corresponding author: Randa Abdelmasih, randa.m.abdelmasih@gmail.com

\begin{abstract}
Cerebrovascular diseases appear to be one of the most serious complications of coronavirus disease 2019 (COVID-19). In this report, we present a case of a 38-year-old male with a past medical history significant only for hypertension, who presented to the emergency department (ED) with confusion and multiple focal neurologic deficits. Brain imaging showed acute multiple cerebral watershed infarctions. Upon further investigation and laboratory workup, the hypercoagulability and vasculitis panels were found to be negative, and other differential diagnoses were ruled out. In light of a number of emerging reports of COVID-19related ischemic stroke, our patient was also screened for the disease, and surprisingly the test came back positive. We believe this case report will highlight the importance of conducting neurological examinations in COVID-19 patients, since timely workup and prompt interventions may reduce morbidity and mortality.
\end{abstract}

Review began 05/10/2021 Review ended 05/12/2021 Published 05/19/2021

(๑) Copyright 2021

Abdelmasih et al. This is an open access article distributed under the terms of the Creative Commons Attribution License CC-BY 4.0., which permits unrestricted use, distribution, and reproduction in any medium, provided the original author and source are credited.
Categories: Internal Medicine, Neurology, Infectious Disease

Keywords: covid 19, stroke, weakness, neurological deficit

\section{Introduction}

The novel coronavirus disease 2019 (COVID-19) pandemic has been regarded as the most severe global public health crisis since the influenza outbreak in 1918. Apart from its various common manifestations, it has also been associated with hypercoagulability that leads to life-threatening cardiovascular and neurovascular complications. The pathogenesis of this predisposition is not well understood, but one of its consequences is an increased risk of stroke. Patients with patent foramen ovale (PFO), atrial fibrillation, previous history of stroke, and elderly patients with uncontrolled hypertension are probably at a higher risk of developing stroke due to COVID-19. We present a rare case of acute multiple cerebral watershed infarctions in a COVID-19 patient who did not present with any of the aforementioned risk factors. Our main objective is to highlight the clinical presentation and the proposed mechanisms of neurovascular involvement in COVID-19.

\section{Case Presentation}

A 38-year-old obese male with a past medical history of hypertension presented to the hospital with confusion, left-sided upper and lower extremity weakness, right lower extremity weakness, and right-gaze preference. CT scan and MRI of the brain demonstrated multiple patchy small areas of acute ischemic infarcts bilaterally consistent with watershed infarcts (Figures 1,2). An echocardiogram with bubble study was negative for PFO. Lower extremity venous Doppler ultrasound was negative for deep venous thrombosis (DVT). Telemetry did not record any arrhythmias. Laboratory workup was negative for autoimmune diseases, hypercoagulable states, and vasculitis including antiphospholipid antibodies, lupus anticoagulants, antineutrophil cytoplasmic antibodies, anti-nuclear antibodies, anti-factor Xa, anti-thrombin, protein S and C, and rapid plasma regain. The patient was screened for COVID-19 in the context of increased reports of stroke in young patients nationwide, and surprisingly, the test was positive. Subsequently, aspirin, clopidogrel, high-intensity statins, and enoxaparin for DVT prophylaxis were initiated. 


\section{Cureus}

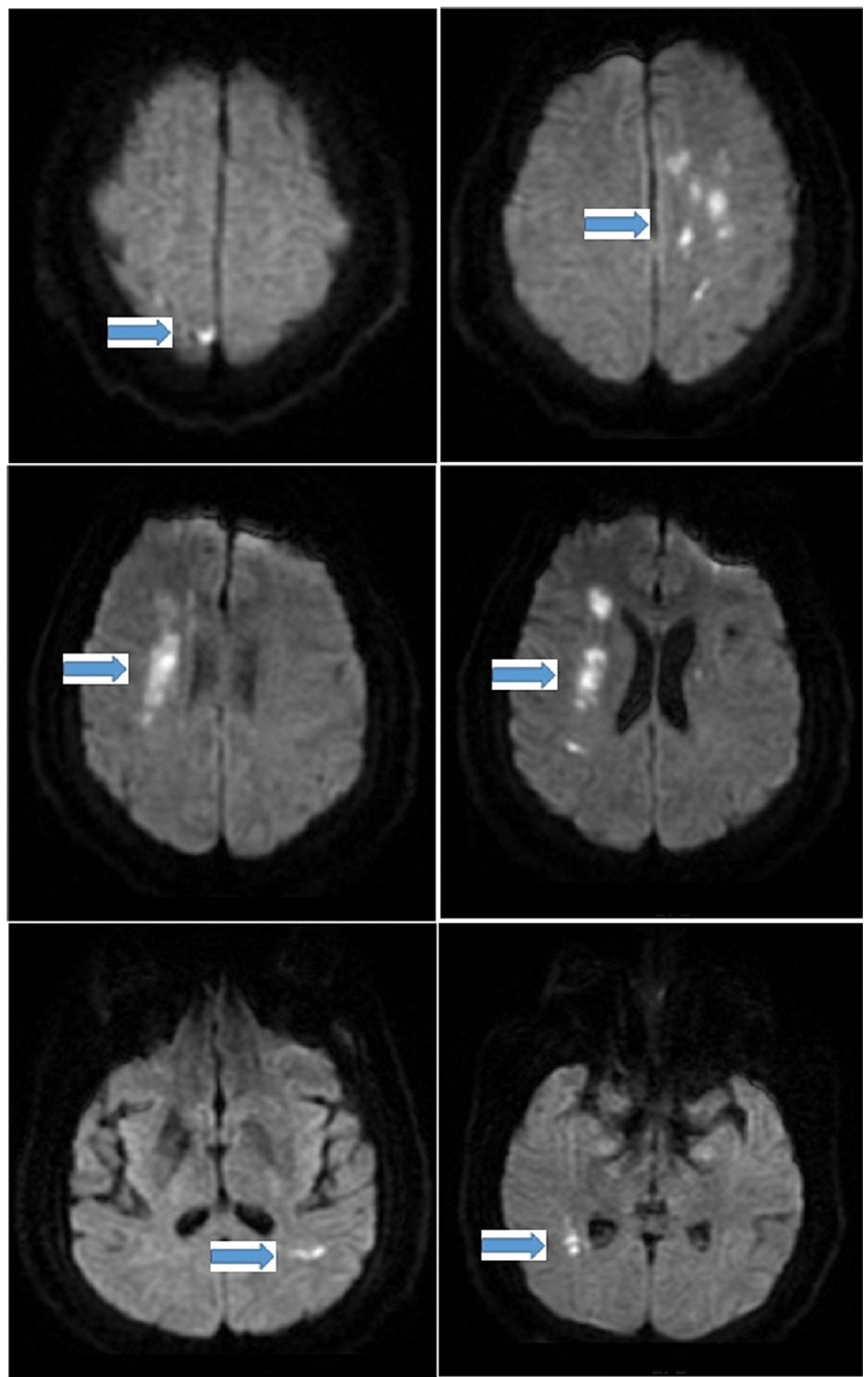

FIGURE 1: MRI brain showing multiple bilateral patchy small areas of acute infarctions related to watershed distribution (arrows)

MRI: magnetic resonance imaging 


\section{Cureus}

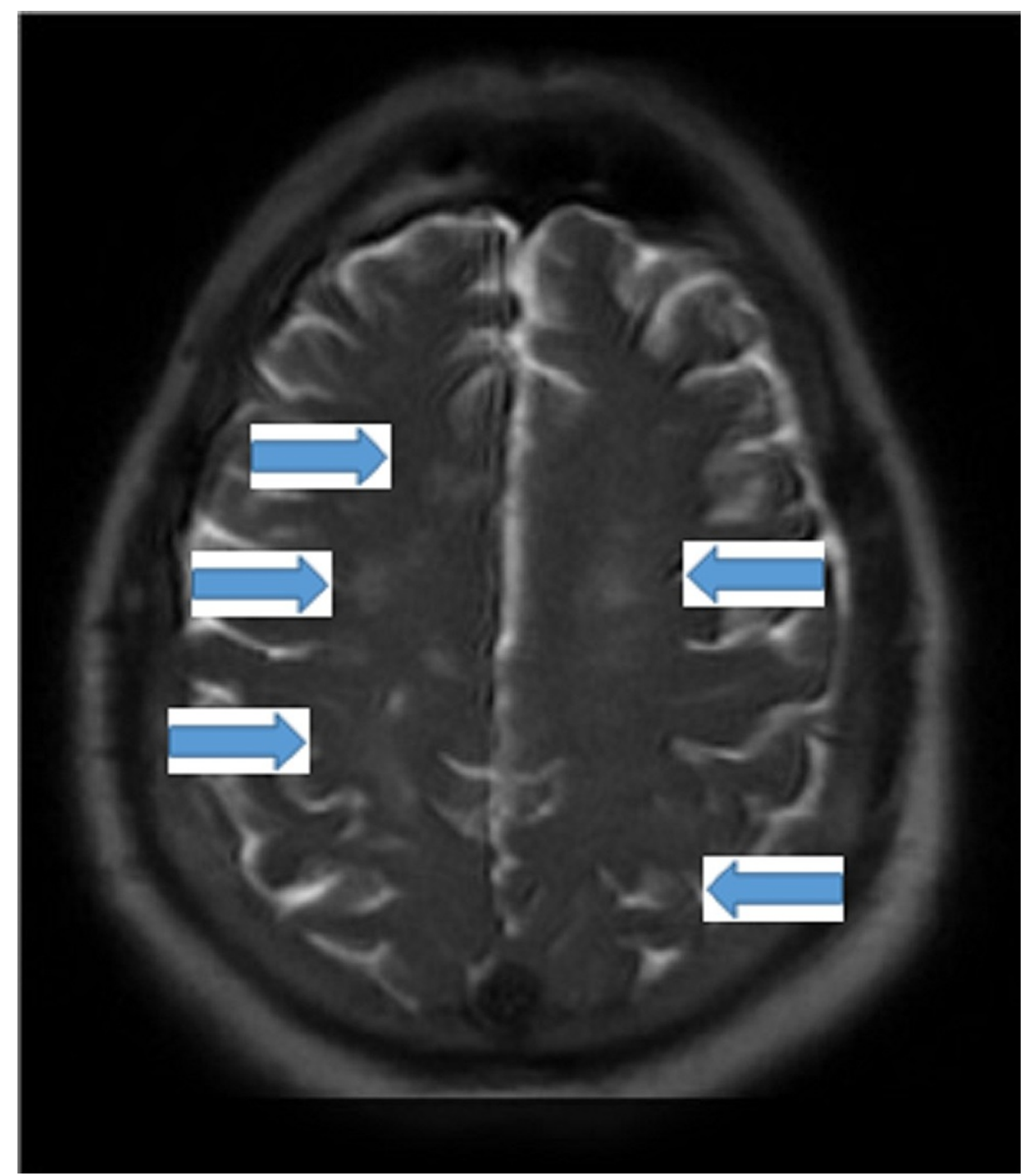

FIGURE 2: MRI brain showing multiple bilateral patchy acute infarctions (arrows)

MRI: magnetic resonance imaging

\section{Discussion}

COVID-19 has turned into a major healthcare emergency of global concern. Evidence increasingly shows that it is not always confined to the respiratory tract but can also induce neurologic diseases with associated increased risk of stroke in young patients [1,2]. The reported incidence of stroke in COVID-19-positive patients is about 1-6\% [3]. Although not completely understood, the plausible proposed mechanism of cerebrovascular disease in COVID-19 includes viral neurotropism, coagulopathy from systemic inflammation and cytokine storm, and endothelial dysfunction leading to angiopathic thrombosis. There have been a handful of cases linking COVID-19 to large vessel macrothrombosis and antiphospholipid syndrome [4]. We reported an even more unusual case that presented with multiple focal neurologic symptoms and was found to have watershed infarctions secondary to COVID-19 with only mild respiratory symptoms, and without typical vascular risk factors, PFO or arrhythmia, after excluding autoimmune diseases, hypercoagulability diseases, and vasculitis; this report highlights the importance of suspecting this devastating disease in an otherwise healthy young patient who presents with focal neurological symptoms.

\section{Conclusions}

In COVID-19 patients with mild respiratory symptoms, a low threshold for investigation for stroke should be adopted if any new neurological symptoms are observed.

\section{Additional Information}




\section{Cureus}

\section{Disclosures}

Human subjects: Consent was obtained or waived by all participants in this study. Conflicts of interest: In compliance with the ICMJE uniform disclosure form, all authors declare the following: Payment/services info: All authors have declared that no financial support was received from any organization for the submitted work. Financial relationships: All authors have declared that they have no financial relationships at present or within the previous three years with any organizations that might have an interest in the submitted work. Other relationships: All authors have declared that there are no other relationships or activities that could appear to have influenced the submitted work.

\section{References}

1. Wijeratne T, Sales C, Karimi L, Crewther SG: Acute ischemic stroke in COVID-19: a case-based systematic review. Front Neurol. 2020, 11:1031. 10.3389/fneur.2020.01031

2. Elkhider H, Ibrahim F, Sharma R, et al.: COVID-19 and stroke, a case series and review of literature . Brain Behav Immun Health. 2020, 9:100172. 10.1016/j.bbih.2020.100172

3. Oxley TJ, Mocco J, Majidi S, et al.: Large-vessel stroke as a presenting feature of covid-19 in the young . N Engl J Med. 2020, 382:e60. 10.1056/NEJMc2009787

4. Mohamed IZB, Balson L, Madathil S: Massive bilateral stroke in a COVID-19 patient . BMJ Case Rep. 2020, 13:e236254. 10.1136/bcr-2020-236254 